\title{
INVARIANT SUBMANIFOLDS FOR AFFINE CONTROL SYSTEMS
}

\author{
CHONG-KYU HAN AND HYESEON KIM
}

\begin{abstract}
Given an affine control system $\dot{\mathbf{x}}=f(\mathbf{x})+\sum_{j=1}^{m} g_{j}(\mathbf{x}) u_{j}$ we present a method of construction of submanifolds that are invariant under controls assuming that the linear span of $f, g_{1}, \ldots, g_{m}$ has constant rank. We use the method of reduction of Pfaffian systems to a largest integrable subsystem and finding the first integrals and the generalized first integrals for the vector fields $f$ and $g_{j}$ 's.
\end{abstract}

\section{Introduction And the Statement of the MAin RESUlts}

Let $M$ be a connected smooth $\left(C^{\infty}\right)$ manifold of dimension $n$ and $\mathcal{U}$ be a set of admissible controls $u:[0, \infty) \rightarrow \mathbb{R}^{m}, m \leqslant n$. We consider an affine control system, which is a system of differential equations of the form:

$$
\dot{x}:=\frac{d x}{d t}=f(x)+\sum_{j=1}^{m} g_{j}(x) u_{j},
$$

where $x=\left(x_{1}, \ldots, x_{n}\right)$ are local coordinates of $M, f$ and $g_{1}, \ldots, g_{m}$ are smooth vector fields on $M$, and $u=\left(u_{1}, \ldots, u_{m}\right) \in \mathcal{U}$. Here the control $u(t)$ can be chosen variously, for instance, to be piecewise continuous, measurable, smooth, and so forth. In this paper we shall assume that $\mathcal{U}$ is the set of all piecewise constant functions with finitely many discontinuities.

Our viewpoint is local, thus $M$ can be regarded throughout this paper as an open ball of $\mathbb{R}^{n}$ centered at a reference point. For a point $p \in M$ let $x(t)$ be the solution of

$$
\left\{\begin{array}{l}
\dot{x}=f(x)+\sum_{j=1}^{m} g_{j}(x) u_{j}(t), \\
x(0)=p .
\end{array}\right.
$$

The solution $x(t)$ is continuous, piecewise smooth and uniquely determined by the choice of $p$ and $u(t)$, which we shall denote by $\Gamma(p, t, u)$.

2010 Mathematics Subject Classification. Primary 57R27, 58A17, 93B05; Secondary $37 \mathrm{C} 10,93 \mathrm{C} 15$.

Key words and phrases. affine control system, orbits, invariant submanifolds, generalized first integrals, controllability. 
Definition 1.1. A submanifold $N$ of $M$ is said to be invariant under controls of (1.1) if $x_{0} \in N$ implies that $\Gamma\left(x_{0}, t, u\right) \in N$ for all possible choices of $u \in \mathcal{U}$ and for all $t \geqslant 0$.

The purpose of this paper is to present a systematic description and a method of construction of invariant submanifolds for (1.1). By a distribution $\mathcal{D}$ of rank $k$ we shall mean a smooth sub-bundle $\mathcal{D}$ of fibre dimension $k$ of the tangent bundle $T M$. A smooth real-valued function $\rho$ is called a first integral of $\mathcal{D}$ if

$$
X \rho=0
$$

for any section $X$ of $\mathcal{D}$. A distribution $\mathcal{D}$ is said to be integrable if

$$
[\mathcal{D}, \mathcal{D}] \subset \mathcal{D}
$$

which means that for any sections $X$ and $Y$ of $\mathcal{D}$ their Lie bracket $[X, Y]$ is a section of $\mathcal{D}$. A system $\rho=\left(\rho^{1}, \ldots, \rho^{d}\right)$ of smooth real-valued functions on $M$ is said to be non-degenerate if

$$
d \rho^{1} \wedge \cdots \wedge d \rho^{d} \neq 0 \text { on a neighborhood of }\{\rho=0\} .
$$

Theorem 1.2 (Frobenius). Let $M$ be a smooth manifold of dimension $n$ and $\mathcal{D}$ a distribution of rank $k$. If $\mathcal{D}$ is integrable, then there exists a nondegenerate system of $n-k$ first integrals $\rho=\left(\rho^{1}, \ldots, \rho^{n-k}\right)$.

Given a distribution $\mathcal{D}$ of rank $k \geqslant 2$, (1.2) is over-determined, and there are no solutions generically. Theorem 1.2 is an extreme case that (1.2) has $(n-k)$ independent solutions. This is the largest possible dimension for the solution space. If $\mathcal{D}$ is not integrable one might construct an integrable distribution of the smallest rank that contains $\mathcal{D}$ as a sub-bundle as follows: Set

$$
\mathcal{D}^{(0)}=\mathcal{D}
$$

and for each $j=1,2, \ldots$,

$$
\mathcal{D}^{(j)}=\mathcal{D}^{(j-1)}+\left[\mathcal{D}^{(j-1)}, \mathcal{D}^{(j-1)}\right]
$$

assuming $\mathcal{D}^{(j-1)}$ and $\mathcal{D}^{(j)}$ have constant rank. Let $\nu$ be the smallest nonnegative integer such that

$$
\mathcal{D}^{(\nu)}=\mathcal{D}^{(\nu+1)}
$$

We call the sequence

$$
\mathcal{D}=\mathcal{D}^{(0)} \subset \mathcal{D}^{(1)} \subset \cdots \subset \mathcal{D}^{(\nu)}
$$

the derived flag of $D$. Generically, this sequence ends up with the whole tangent bundle, that is, $\mathcal{D}^{(\nu)}=T M$. 
Definition 1.3. Let $\mathcal{D}^{(\nu)}$ be as in (1.3). If $\mathcal{D}^{(\nu)}$ has rank $\ell$, then $\mathcal{D}$ is said to be of type $(\nu, \ell)$.

Notice that $\mathcal{D}^{(\nu)}$ is the smallest integrable distribution that contains $\mathcal{D}$ as a sub-bundle. Theorem 1.2 is the case that $\mathcal{D}$ has type $(0, k)$. Observe that if $\mathcal{D}$ has type $(\nu, \ell)$ there exists a non-degenerate system of $n-\ell$ first integrals since $\mathcal{D}^{(\nu)}$ is integrable in the sense of Frobenius. This implies that $M$ is foliated by $(n-\ell)$-parameter family of invariant submanifolds of $\mathcal{D}$ (see Theorem 1.8). To discuss the existence of isolated (zero-parameter family) invariant submanifolds we need the notion of the generalized first integral, which was first introduced in [2].

Definition 1.4. Let $\mathcal{D}$ be a distribution. A non-degenerate set $\rho=\left(\rho^{1}, \ldots, \rho^{d}\right)$ of smooth real-valued functions is called a system of generalized first integrals of $\mathcal{D}$ if (1.2) holds on the zero locus of $\rho$, that is, for any section $X$ of $\mathcal{D}$,

$$
(X \rho)(x)=0, \quad \text { for all } x \in M \quad \text { with } \rho(x)=0 .
$$

In this paper, we focus our attention to the affine control system (1.1) under an assumption that the linear span of $f, g_{1}, \ldots, g_{m}$ has constant rank.

Now we recall some basics of non-linear control systems. For other definitions and theorems we refer the readers to our basic references [1, 12, 13, 14]. Consider the set of points that are reachable from a point in some nonnegative time. A point $q \in M$ is said to be reachable from $p$ if $q=\Gamma(p, t, u)$, for some $u \in \mathcal{U}$ and for some $t \geqslant 0$. The reachable set $\mathcal{R}_{p}$ of the control system (1.1) from a point $p \in M$ is a subset of $M$ defined by

$$
\mathcal{R}_{p}=\{\Gamma(p, t, u): t \geqslant 0, u \in \mathcal{U}\} .
$$

The control system (1.1) is said to be controllable from $p \in M$ if

$$
\mathcal{R}_{p}=M
$$

and it is called controllable if (1.5) holds for every $p \in M$. We define the orbit $\mathcal{O}_{p}$ of the control system (1.1) through a point $p \in M$ to be the set of points $q \in M$ such that either $q$ is reachable from $p$ or $p$ is reachable from $q$.

The term $f(x)$ in (1.1) is called the drift. It is obvious that for affine control systems without drift we have

$$
\mathcal{R}_{p}=\mathcal{O}_{p}
$$

for each $p \in M$. We observe also that the Nagano-Sussmann orbit theorem [1] holds for the affine control system (1.1) regardless of the drift, that is,

Theorem 1.5. $\mathcal{O}_{p}$ is a connected immersed submanifold of $M$. 
Let $\mathcal{G}$ be the Lie algebra of vector fields generated by $g_{1}, \ldots, g_{m}$. Consider the vector space $\mathcal{G}(p) \subset T_{p} M$, which is the linear span at $p \in M$ of the left iterated Lie brackets

$$
\left[g_{i_{1}},\left[g_{i_{2}}, \cdots,\left[g_{i_{k-1}}, g_{i_{k}}\right] \cdots\right]\right]
$$

of the vector fields $g_{1}, \ldots, g_{m}$. Then we have

Theorem 1.6 (Rashevsky-Chow theorem, [1]). Let $M$ and $\mathcal{G}$ be as above. If $M$ is connected and $\mathcal{G}(p)=T_{p} M$ for a point $p \in M$, then $\mathcal{O}_{p}$ contains an open neighborhood of $p$.

Now we state our main results as follows:

Theorem 1.7. Suppose that the vector fields $f, g_{1}, \ldots, g_{m}$ span a subspace $\mathcal{D}$ of constant rank. Let $N$ be a submanifold defined as the common zero set of real-valued functions $\rho=\left(\rho^{1}, \ldots, \rho^{d}\right)$ with $d \rho^{1} \wedge \cdots \wedge d \rho^{d} \neq 0$. Then $N$ is invariant under controls of (1.1) if and only if $\rho$ is a system of generalized first integrals of $\mathcal{D}$.

Theorem 1.8. Let $\mathcal{D}$ be as in Theorem 1.7. If $\mathcal{D}$ has type $(\nu, \ell), 1 \leqslant$ $\ell \leqslant n$, then $M$ is foliated by $\ell$-dimensional submanifolds that are invariant under controls of (1.1). This type condition is given by a system of partial differential equations of order $(\nu+1)$ for $f$ and $g_{j}, j=1, \ldots, m$.

Given a control system proving its controllability is primarily important in geometric control theory, see [10]. In this paper we discuss its "noncontrollability' determining the existence of invariant submanifolds. As a possible application we would be able to design control systems with prescribed invariant submanifolds. In $\$ 3$ we construct the derived flag of $\mathcal{D}$ and find the generalized first integrals by using various generalizations of the Frobenius theorem on involutivity (cf. [10] and [11]). \$4 is devoted to examples of invariant submanifolds.

\section{Proof of the MAIN RESUlts}

2.1. Proof of Theorem 1.7. Suppose that $\rho=\left(\rho^{1}, \cdots, \rho^{d}\right)$ is a nondegenerate system of real-valued functions whose common zero set $N$ is invariant under controls of (1.1). To show that $\rho$ is a generalized first integral it suffices to show that

$$
(f \rho)(x)=\left(g_{j} \rho\right)(x)=0, j=1, \ldots, m, \text { for all } x \in N,
$$


since $f, g_{1}, \ldots, g_{m}$ span $\mathcal{D}$. Let us fix a point $p \in N$. If we choose control $u(t)=0$, to be constantly zero, the trajectory $x(t)$ with $x(0)=p$ is the integral curve of $f$ through $p$. Since $N$ is invariant under controls of (1.1), we have by the chain rule

$$
0=\frac{d}{d t} \rho(x(t))=(\dot{x} \rho)(x(t))=(f \rho)(x(t)) .
$$

Substituting $t=0$ in (2.2), we obtain

$$
(f \rho)(p)=0 .
$$

Now for each $j=1, \ldots, m$, let $u(t)=\left(u_{1}(t), \ldots, u_{m}(t)\right) \in \mathcal{U}$ defined by $u_{j}(t)=1$ and $u_{i}(t)=0$ for all $i \neq j$. Then there exists a unique trajectory $y(t)$ in some finite time interval with $y(0)=p$ which is the integral curve of

$$
\dot{x}=f(x)+g_{j}(x) .
$$

In a similar fashion to (2.2) and (2.3), one can deduce that

$$
\left(\left(f+g_{j}\right) \rho\right)(p)=0 .
$$

From (2.3) and (2.4) we have

$$
\left(g_{j} \rho\right)(p)=0 .
$$

Since $p$ was arbitrary, (2.3) and (2.5) prove the assertion (2.1).

Conversely, suppose that $\rho=\left(\rho^{1}, \ldots, \rho^{d}\right)$ is a non-degenerate system of generalized first integrals of $\mathcal{D}$. Let $N$ be the zero locus of $\rho$. We now fix a point $p \in N$. Consider first a constant control $u_{c}(t)=c, c=\left(c_{1}, \ldots, c_{m}\right)$. Since $f$ and $g_{j}$ are tangent to $N$, we see that

$$
f+\sum_{j=1}^{m} c_{j} g_{j}
$$

is tangent to $N$. This implies that $\Gamma\left(p, t, u_{c}\right) \in N$, that is, $\rho(x(t))=0$. Now for a piecewise constant control $u=u(t)$, we apply the same argument to each piece to obtain $\Gamma(p, t, u(t)) \in N$. Therefore, the orbit of $p$ is contained in $N$, which implies that $N$ is invariant under the controls of (1.1).

2.2. Proof of Theorem 1.8. Suppose that a smooth real-valued function $\rho$ is a first integral of $\mathcal{D}$ and that $Z$ is a section of $[\mathcal{D}, \mathcal{D}]$, namely, $Z=[X, Y]$, for some sections $X, Y$ of $\mathcal{D}$. Then

$$
Z \rho=(X Y-Y X) \rho=0 .
$$

This implies that $\rho$ is a first integral of $\mathcal{D}^{(1)}$. By induction, $\rho$ is a first integral of $\mathcal{D}^{(\nu)}$. Since $\mathcal{D}^{(\nu)}$ is integrable and has rank $\ell$, there exists a non-degenerate system $\rho=\left(\rho^{1}, \ldots, \rho^{n-\ell}\right)$ of first integrals by Theorem 1.2. Thus $M$ is 
foliated by integral manifolds $\rho=$ constant of $\mathcal{D}^{(\nu)}$, where the dimension of each leaf is $\ell$. Moreover, Theorem 1.7 implies that each leaf $N$ is an invariant submanifold under controls of (1.1). Notice that the condition $\left[\mathcal{D}^{(\nu)}, \mathcal{D}^{(\nu)}\right] \subset \mathcal{D}^{(\nu)}$ involves the derivatives of $f, g_{j}, j=1, \ldots, m$, up to order $\nu+1$. This will be shown more explicitly in the dual arguments of \$3 , where we construct the derived flag of the associated Pfaffian system.

\section{Construction of the Derived Flag AND the Generalized}

\section{FIRST INTEGRALS}

This section is mainly concerned with the systematic construction of the derived flag and the generalized first integrals of a distribution, which are defined in $\$ 1$. For the sake of computation, we use the exterior differentiation and algebraic operations on differential ideals instead of vector fields and their brackets. This approach has been used also in [2, 8, 9] for the cases of real vector fields and in [5, 6, 7] for the cases of complex vector fields. The notion of type for Pfaffian system (Definition 3.2) is due to R. B. Gardner [4].

Let $M$ be a smooth real manifold of dimension $n$. Let $\Omega^{0}$ be the ring of smooth real-valued functions on $M$ and $\Omega^{k}(1 \leqslant k \leqslant n)$ the module over $\Omega^{0}$ of smooth $k$-forms on $M$ with smooth real coefficients. Then $\Omega^{*}:=\oplus_{k=0}^{n} \Omega^{k}$ is the exterior algebra equipped with operations of the wedge product $\wedge$ and the exterior differentiation $d$. Our standard reference for this section is [3].

Definition 3.1. A subalgebra $\mathcal{I}$ of $\Omega^{*}$ is called an algebraic ideal if

(i) $\mathcal{I} \wedge \Omega^{*} \subset \mathcal{I}$;

(ii) if $\phi=\sum_{k=0}^{n} \phi_{k} \in \mathcal{I}, \phi_{k} \in \Omega^{k}$, then each $\phi_{k}$ is in $\mathcal{I}$ (homogeneity condition).

Note that the homogeneity condition implies that $\mathcal{I}$ is a two-sided ideal, that is,

$$
\Omega^{*} \wedge \mathcal{I} \subset \mathcal{I}
$$

Let $\psi=\left(\psi^{1}, \ldots, \psi^{l}\right)$ be a system of smooth differential forms of any degree. We denote by $(\psi)$ the algebraic ideal generated by $\psi$. Then each element of $(\psi)$ has the form

$$
\sum_{k=1}^{l} \xi^{k} \wedge \psi^{k}
$$

for some $\xi^{k} \in \Omega^{*}$. For $\alpha, \beta \in \Omega^{*}$, if $\alpha-\beta \in(\psi)$ we write $\alpha \equiv \beta$, $\bmod (\psi)$. 
Now let $X_{1}, \ldots, X_{p}$ be linearly independent smooth vector fields on $M$ and $\mathcal{D}$ a distribution generated by them. Consider a system of independent smooth 1-forms $\theta:=\left(\theta^{1}, \ldots, \theta^{s}\right), s+p=n$, on $M$ which annihilates $X_{1}, \ldots, X_{p}$. We denote by $I$ the sub-bundle of the cotangent bundle $T^{*} M$ generated by $\theta$. Now we fix notations: For any sub-bundle $I$ of $T^{*} M$ we denote by $\underline{I}$ the $\Omega^{0}$-module of smooth sections of $I$ and by $(I)$ the algebraic ideal of $\Omega^{*}$ generated by the smooth sections of $I$. Then the Frobenius integrability for $\mathcal{D}$ becomes

$$
d \underline{\mathrm{I}} \subset(I) .
$$

A set of real-valued functions $\rho=\left(\rho^{1}, \ldots, \rho^{d}\right)$ is a first integral if

$$
d \rho \in \underline{\mathrm{I}} .
$$

Now we consider the composition of the exterior differentiation $d: \underline{I} \rightarrow \Omega^{*}$ and the natural projection $\pi: \Omega^{*} \rightarrow \Omega^{*} /(I)$ :

$$
\underline{\mathrm{I}} \stackrel{d}{\rightarrow} \Omega^{2} \stackrel{\pi}{\rightarrow} \Omega^{2} /(I)
$$

Then $\delta:=\pi \circ d$ is a module homomorphism. We define a sub-module $\underline{\mathrm{I}}^{(1)}$ of I by setting

$$
\underline{\mathrm{I}}^{(1)}=\operatorname{ker} \delta .
$$

Assuming that $\underline{I}^{(1)}$ has constant rank, $I^{(1)} \subset T^{*} M$ is now a sub-bundle of $T^{*} M$ which we call the first derived system of $I$. Then the sequence

$$
0 \rightarrow \underline{\mathrm{I}}^{(1)} \rightarrow \underline{\mathrm{I}} \stackrel{\delta}{\rightarrow} d \underline{\mathrm{I}} /(I) \rightarrow 0
$$

is exact. Assuming that $\underline{I}^{(k-1)}$ has constant rank on $M$, we define inductively the $k$-th derived system $I^{(k)}$ of $I$ by the exactness of

$$
0 \rightarrow \underline{\mathrm{I}}^{(k)} \rightarrow \underline{\mathrm{I}}^{(k-1)} \stackrel{\delta}{\rightarrow} d \underline{\mathrm{I}}^{(k-1)} /\left(I^{(k-1)}\right) \rightarrow 0 .
$$

By setting $\nu$ to be the smallest non-negative integer such that

$$
I^{(\nu)}=I^{(\nu+1)}
$$

we obtain a sequence

$$
I=I^{(0)} \supset I^{(1)} \supset \cdots \supset I^{(\nu-1)} \supset I^{(\nu)},
$$

which we shall call the derived flag of $I$. Notice that $I^{(\nu)}$ is the largest subbundle of $I$ that satisfies the Frobenius integrability (3.1). Moreover, by using the formula

$$
d \varphi(X, Y)=X \varphi(Y)-Y \varphi(X)-\varphi([X, Y])
$$


for all 1 -form $\varphi$ and all smooth vector fields $X$ and $Y$, it is easy to see that for each $k=0,1, \ldots, \nu$,

$$
\mathcal{D}^{(k)^{\perp}}=\underline{\mathrm{I}}^{(k)} .
$$

Definition 3.2. Let $I$ and $I^{(\nu)}$ be as above. If $I^{(\nu)}$ has rank $q$, then $I$ is said to be of type $(\nu, q)$.

Compare this to Definition 1.3 and observe that $I$ has type $(\nu, q)$ if and only if $\mathcal{D}$ has type $(\nu, n-q)$ by (3.3). We observe also that (3.2) implies $d \rho \in I^{(1)}$ and inductively $d \rho \in I^{(\nu)}$. Thus we have the following proposition, which is a basic observation of [4].

Proposition 3.3. A real-valued function $\rho$ is a first integral if and only if $d \rho \in I^{(\nu)}$. Therefore, if I has type $(\nu, q)$ then there is a q-parameter family of non-degenerate first integrals.

Now the defining property (1.4) of the generalized first integral states as follows:

Proposition 3.4. On a smooth manifold $M^{n}$, let $\mathcal{D}$ be a distribution spanned by vector fields $X_{1}, \ldots, X_{p}$, and $\theta=\left(\theta^{1}, \ldots, \theta^{s}\right), p+s=n$, be 1-forms that annihilate $\mathcal{D}$. Let $\rho=\left(\rho^{1}, \ldots, \rho^{d}\right), d \leqslant s$, be a non-degenerate set of smooth real-valued functions. Then $\rho$ is a system of generalized first integrals of $\mathcal{D}$ if and only if

$$
d \rho^{\mu} \in(\rho, \theta), \mu=1, \ldots, d \text {. }
$$

Now let $\omega^{1}, \ldots, \omega^{p}$ be a set of closed smooth 1 -forms that completes $\theta$ to a local coframe

$$
\left(\theta^{1}, \ldots, \theta^{s}, \omega^{1}, \ldots, \omega^{p}\right)
$$

of $M$. Set

$$
d \theta^{l} \equiv \sum_{j<k} T_{j, k}^{l} \omega^{j} \wedge \omega^{k}, \bmod (\theta), l=1, \ldots, s .
$$

In matrices, (3.6) is written as

$$
\left[\begin{array}{c}
d \theta^{1} \\
\vdots \\
d \theta^{s}
\end{array}\right] \equiv \underbrace{\left[\begin{array}{cccc}
T_{1,2}^{1} & T_{1,3}^{1} & \cdots & T_{p-1, p}^{1} \\
\vdots & & & \vdots \\
T_{1,2}^{s} & T_{1,3}^{s} & \cdots & T_{p-1, p}^{s}
\end{array}\right]}_{\mathcal{T}}\left[\begin{array}{c}
\omega^{1} \wedge \omega^{2} \\
\omega^{1} \wedge \omega^{3} \\
\vdots \\
\omega^{p-1} \wedge \omega^{p}
\end{array}\right], \bmod (\theta) .
$$

$\mathcal{T}$ in (3.7) is a matrix of size $s \times\left(\begin{array}{l}p \\ 2\end{array}\right)$, called the torsion matrix with respect to the coframe (3.5). If $\mathcal{T} \equiv 0$, this is the case of the Frobenius theorem. If 
$\mathcal{T} \not \equiv 0$, then we find generators of $I^{(\nu)}$ as follows: Suppose that

$$
\phi:=\sum_{\gamma=1}^{s} a_{\gamma} \theta^{\gamma} \in I^{(1)}=\operatorname{ker} \delta .
$$

Then we have

$$
d \phi \equiv \sum_{\gamma=1}^{s} a_{\gamma} d \theta^{\gamma} \equiv 0, \bmod (\theta) .
$$

Substituting (3.6) for $d \theta^{\gamma}$ in (3.9), it follows from the independence of $\omega^{k} \wedge \omega^{l}$ in $\Omega^{2} /(I)$ that the row vector $\left(a_{1}, \ldots, a_{s}\right)$ belongs to the left null-space of $\mathcal{T}$. By finding the generators of the left null-space of $\mathcal{T}$ we obtain a set of generators (3.8) of $I^{(1)}$. Then by the linear algebra of the torsion matrix for the Pfaffian system $I^{(1)}$, we obtain the generators of $I^{(2)}$, and for $I^{(\nu)}$ by induction.

We use similar arguments for the generalized first integrals. Suppose that $\rho=\left(\rho^{1}, \ldots, \rho^{d}\right), d \leqslant s$, is a non-degenerate system of generalized first integrals of $\mathcal{D}$. Then, for each $\mu, 1 \leqslant \mu \leqslant d, d \rho^{\mu} \in(\rho, \theta)$, namely

$$
d \rho^{\mu}=\sum_{\lambda=1}^{d} \rho^{\lambda} \psi_{\lambda}^{\mu}+\sum_{\gamma=1}^{s} b_{\gamma}^{\mu} \theta^{\gamma}
$$

for some smooth 1-forms $\psi_{\lambda}^{\mu}$ and some smooth functions $b_{\gamma}^{\mu}$. Applying $d$ to (3.10), we have

$$
0 \equiv \sum_{\gamma=1}^{s} b_{\gamma}^{\mu} d \theta^{\gamma}, \bmod (\rho, \theta), \mu=1, \ldots, d .
$$

Consider the matrix of coefficients

$$
\mathcal{B}=\left[\begin{array}{ccc}
b_{1}^{1} & \cdots & b_{s}^{1} \\
\vdots & & \vdots \\
b_{1}^{d} & \cdots & b_{s}^{d}
\end{array}\right]
$$

In matrices (3.11) can be written as

$$
\left[\begin{array}{c}
0 \\
0 \\
\vdots \\
0
\end{array}\right] \equiv \mathcal{B T}\left[\begin{array}{c}
\omega^{1} \wedge \omega^{2} \\
\omega^{1} \wedge \omega^{3} \\
\vdots \\
\omega^{p-1} \wedge \omega^{p}
\end{array}\right], \bmod (\rho, \theta) .
$$

Since $\omega^{k} \wedge \omega^{l}$ are independent 2-forms we have

$$
\mathcal{B} \mathcal{T} \equiv 0, \bmod (\rho) .
$$

Moreover, since $\left(\rho^{1}, \ldots, \rho^{d}\right)$ are non-degenerate $\mathcal{B}$ has maximal rank $d$ on the zero locus of $\rho$, which implies that $\operatorname{rank} \mathcal{T} \leqslant s-d$ on the zero locus of $\rho$. Thus we observed that any square sub-matrix of $\mathcal{T}$ of size $s-d+1$ has determinant zero on the zero locus of $\rho$. Then we factor those determinants, 
find non-degenerate functions that generate all those determinants, which are the candidates to be the generalized first integral $\rho$. Finally, if thus found $\rho$ satisfies (3.4), then $\rho$ is the desired system of generalized first integrals.

\section{EXAmples}

The examples we present in this section are rather artificial, devised simply to show how our method works.

Example 4.1 (a single invariant submanifold). Consider an affine control system on $\mathbb{R}^{3}=\{(x, y, z)\}$ given by

$$
\left[\begin{array}{c}
\dot{x} \\
\dot{y} \\
\dot{z}
\end{array}\right]=\underbrace{\left[\begin{array}{c}
1 \\
y \\
0
\end{array}\right]}_{g_{1}} u_{1}+\underbrace{\left[\begin{array}{c}
0 \\
1 \\
x z
\end{array}\right]}_{g_{2}} u_{2}:=g_{1} u_{1}+g_{2} u_{2},
$$

where $\mathbb{R}^{3}=\{(x, y, z)\}$ is the state space and $\left(u_{1}, u_{2}\right) \in \mathbb{R}^{2}$ are controls. Then

$$
\theta=x y z d x-x z d y+d z
$$

annihilates $g_{1}$ and $g_{2}$. We take up a coframe $\{\theta, d x, d y\}$. Then with respect to this coframe

$$
d \theta \equiv \underbrace{-z(1+x)}_{\mathcal{T}} d x \wedge d y, \bmod (\theta) .
$$

In this case the torsion is a $1 \times 1$ matrix $\mathcal{T}=-z(1+x)$. Any non-degenerate factor of $T$, in particular, $\rho(x, y, z)=z$ is a candidate for generalized first integral. Now we shall examine the condition (3.4) for $\rho(x, y, z)=z$. Indeed, we have

$$
d \rho=d z=\theta-\rho(x y d x-x d y) \in(\rho, \theta) .
$$

Therefore, $\{z=0\}$ is an invariant submanifold. Since $g_{1}$ and $g_{2}$ are independent on $z=0$ the system (4.1) restricted on $z=0$ is controllable.

Example 4.2 (no invariant submanifolds). We slightly change (4.1) to

$$
\left[\begin{array}{c}
\dot{x} \\
\dot{y} \\
\dot{z}
\end{array}\right]=\underbrace{\left[\begin{array}{c}
1 \\
y \\
0
\end{array}\right]}_{h_{1}} u_{1}+\underbrace{\left[\begin{array}{c}
0 \\
1 \\
x y
\end{array}\right]}_{h_{2}} u_{2}:=h_{1} u_{1}+h_{2} u_{2} .
$$

In the same way as in the previous example we have

$$
\theta=x y^{2} d x-x y d y+d z
$$

so that

$$
d \theta=\underbrace{-y(1+2 x)}_{\text {torsion }} d x \wedge d y
$$


Now $\rho(x, y, z)=y$ is a non-degenerate factor of the torsion, which is unique modulo multiplication by unit. Hence the only candidate to be an invariant submanifold is $y=0$. However,

$$
d \rho=d y \notin(y, \theta)
$$

therefore, there are no invariant submanifolds.

Example 4.3 (foliation by invariant submanifolds, vehicle on a slanted plane). First we describe the motion of a car on a slanted plane as follows: Let $M=\left\{(x, y, z, w): x, y, z \in \mathbb{R}^{3}, w \in S^{1}\right\}$ be the state space of the affine control system

$$
\left[\begin{array}{c}
\dot{x} \\
\dot{y} \\
\dot{z} \\
\dot{w}
\end{array}\right]=\left[\begin{array}{c}
\cos \phi_{0} \cos w \\
\sin w \\
\sin \phi_{0} \cos w \\
0
\end{array}\right] u_{1}+\left[\begin{array}{l}
0 \\
0 \\
0 \\
1
\end{array}\right] u_{2},
$$

where $(x, y, z)$ is the position of the center of mass of the car and $w$ is the angle measured from the first coordinate axis of the slanted plane to the direction of the vehicle. Assuming the slanted plane meets with the $x y$ plane along $y$-axis, the first coordinate line of the slanted plane intersects with $y$-axis perpendicularly, and $\phi_{0}, 0<\phi_{0}<\pi / 2$, is the angle between these two planes. We assume two possible motions of a car: one can drive the car forward and backwards with a fixed linear velocity $u_{1} \in \mathbb{R}$, and turn the car around its center of mass with a fixed angular velocity $u_{2} \in \mathbb{R}$. We note that this control system is a modification of the typical model of a car in $\mathbb{R}^{2} \times S^{1}$ (cf. [1, Example 1.29]). However, we shall work in a more general setting so that $a$ and $b$ are assumed to be any positive real numbers. We consider

$$
\underbrace{\left[\begin{array}{c}
\dot{x} \\
\dot{y} \\
\dot{z} \\
\dot{w}
\end{array}\right]}_{\dot{\mathbf{x}}}=\underbrace{\left[\begin{array}{c}
a \cos w \\
\sin w \\
b \cos w \\
0
\end{array}\right]}_{g_{1}} u_{1}+\underbrace{\left[\begin{array}{c}
0 \\
0 \\
0 \\
1
\end{array}\right]}_{g_{2}} u_{2}:=g_{1} u_{1}+g_{2} u_{2} .
$$

The system of 1 -forms $\theta=\left(\theta^{1}, \theta^{2}\right)$ given by

$$
\begin{aligned}
& \theta^{1}=b d x-a d z \\
& \theta^{2}=b \cos w d y-\sin w d z
\end{aligned}
$$


annihilates $g_{1}$ and $g_{2}$. Let $I:=(\theta)$ be the ideal. Then we have

$$
\begin{aligned}
{\left[\begin{array}{l}
d \theta^{1} \\
d \theta^{2}
\end{array}\right] } & =\left[\begin{array}{c}
0 \\
-b \sin w d w \wedge d y-\cos w d w \wedge d z
\end{array}\right] \\
& \equiv\left[\begin{array}{c}
0 \\
\sec w d z \wedge d w
\end{array}\right], \bmod \left(\theta^{2}\right) .
\end{aligned}
$$

We see that $I$ has type $(1,1)$ and the first derived system $I^{(1)}$ is generated by $\theta^{1}$. The first integral is

$$
\rho(x, y, z, w)=b x-a z,
$$

and therefore, hyperplanes

$$
b x-a z=\text { constant }
$$

are invariant under controls of (4.2). Since $g_{1}, g_{2}$ and

$$
\left[g_{1}, g_{2}\right]=(a \sin w,-\cos w, b \sin w, 0)^{t}
$$

are independent on each hyperplane of (4.3) the control system (4.2) restricted to each of these invariant hyperplanes is controllable by (1.6) and Theorem 1.6.

Example 4.4 (slanted plane with drift). Consider an affine control

$$
\dot{\mathbf{x}}=f(\mathbf{x})+g_{1}(\mathbf{x}) u_{1}+g_{2}(\mathbf{x}) u_{2},
$$

where $\mathbf{x}$ and $g_{1}, g_{2}$ are the same as in (4.2) and $f$ is a drift. The following are obvious:

a) If $f$ is contained in the linear span of $\left(g_{1}, g_{2}\right)$ then $\rho(x, y, z, w)=b x-a z$ is a first integral, and therefore, $M$ is foliated by the invariant submanifolds $\rho=$ constant .

b) If $f$ has a non-zero component only in $y$-direction, then $\rho(x, y, z, w)=$ $b x-a z$ is a first integral because $\rho$ is independent of $y$ variable.

c) For generic choices of $f$ invariant submanifolds do not exist.

A single invariant submanifold, or equivalently, a generalized first integral, occurs only under special assumptions on $f$. For instance, if $f=$ $(\phi, 1,0,0)^{t}$, where $\phi$ is any function that is divisible by $\rho=b x-a z$, then $\rho$ is a generalized first integral, therefore, a single hyperplane $\rho=0$ is invariant under controls of (4.4). This is obvious because $f=(0,1,0,0)^{t}$ on $\rho=0$, so that $f$ has only $y$-directional component. In fact,

$$
\theta=-b \cos w d x+b \phi \cos w d y+(a \cos w-\phi \sin w) d z
$$


annihilates $f, g_{1}$ and $g_{2}$. Since $d \rho=b d x-a d z$ and $\phi$ is divisible by $\rho$, rearranging the right hand side of (4.5) we have

$$
\theta=-\cos w d \rho+\rho \alpha(b \cos w d y-\sin w d z), \quad \text { for some function } \alpha \text {. }
$$

Solving (4.6) for $d \rho$ yields (3.4). Observe also that

(4.7) $d \theta \equiv-\phi \sec w d w \wedge d z+b \cos w d \phi \wedge d y-\sin w d \phi \wedge d z, \quad \bmod (\theta)$.

Since $\phi=\rho \alpha$, for some $\alpha$, (4.7) implies that

$$
d \theta=\rho \wedge \Psi^{1}+d \rho \wedge \Psi^{2}+\theta \wedge \Psi^{3}, \quad \text { for some } \Psi^{j} \in \Omega^{*}, j=1,2,3 .
$$

For the tangent vectors to $\rho=0$ the first two terms of the right hand side are zero and (4.8) implies that $\theta$ is Frobenius integrable on $\rho=0$. But in this particular case, the foliation has a single leaf: the submanifold $\rho=0$ itself.

Acknowledgements. The second author was supported by National Research Foundation of Korea with grant NRF-2015R1A2A2A11001367.

\section{REFERENCES}

[1] A. Agrachev and Y. Sachkov, Control theory from the geometric viewpoint, Encyclopaedia of Mathematical Sciences, Control Theory and Optimization, II, vol. 87, Springer-Verlag, Berlin, 2004.

[2] H. Ahn and C.-K. Han, Invariant submanifolds for systems of vector fields of constant rank, Sci. China Math. 59 (2016), no. 7, 1417-1426.

[3] R. L. Bryant, S. S. Chern, R. B. Gardner, H. L. Goldschmidt and P. A. Griffiths, Exterior differential systems, Mathematical Sciences Research Institute Publications, vol. 18, Springer-Verlag, New-York, 1991.

[4] R. B. Gardner, Invariants of Pfaffian systems, Trans. Amer. Math. Soc. 126 (1967), 514-533.

[5] C.-K. Han and H. Kim, Holomorphic functions on almost complex manifolds, J. Korean Math. Soc. 49 (2012), no. 2, 379-394.

[6] C.-K. Han and K.-H. Lee, Integrable submanifolds in almost complex manifolds, J. Geom. Anal. 20 (2010), no. 1, 177-192.

[7] C.-K. Han and J.-D. Park, Partial integrability of almost complex structures and the existence of solutions of quasilinear Cauchy-Riemann equations, Pacific J. Math. 265 (2013), no. 1, 59-84.

[8] C.-K. Han and J.-D. Park, Quasi-linear systems of PDE of first order with Cauchy data of higher codimensions, J. Math. Anal. Appl. 430 (2015), no. 1, 390-402. 
[9] C.-K. Han and J.-D. Park, Method of characteristics and first integrals for systems of quasi-linear partial differential equations of first order, Sci. China Math. 58 (2015), no. 8, 1665-1676.

[10] A. Isidori, Nonlinear control systems, Springer-Verlag, London, 1995.

[11] A. Isidori, A. J. Krener, C. Gori-Giorgi and S. Monaco, Nonlinear decoupling via feedback: A differential geometric approach, IEEE Transactions on automatic control AC-26 (1981), 331-345.

[12] V. Jurdjevic, Geometric control theory, Cambridge Studies in Advanced Mathematics, vol. 52, Cambridge University Press, Cambridge, 1997.

[13] H. Nijmeijer and A. J. van der Schaft, Nonlinear Dynamical Control Systems, Springer-Verlag, New York-Heidelberg-Berlin, 1990.

[14] H. J. Sussmann, Orbits of families of vector fields and integrability of distributions, Trans. Amer. Math. Soc. 180 (1973), 171-188.

(C.-K. Han) Department of Mathematical Sciences, Seoul National University, 1 Gwanak-ro, Gwanak-Gu, Seoul 08826, Republic of Korea

E-mail address: ckhan@snu.ac.kr

(H. Kim) Research Institute of Mathematics, Seoul National University, 1 Gwanak-ro, Gwanak-gu, Seoul 08826, Republic of Korea

E-mail address: hop222@gmail.com 